\title{
IX Jornada de Ciencia y Tecnología
}

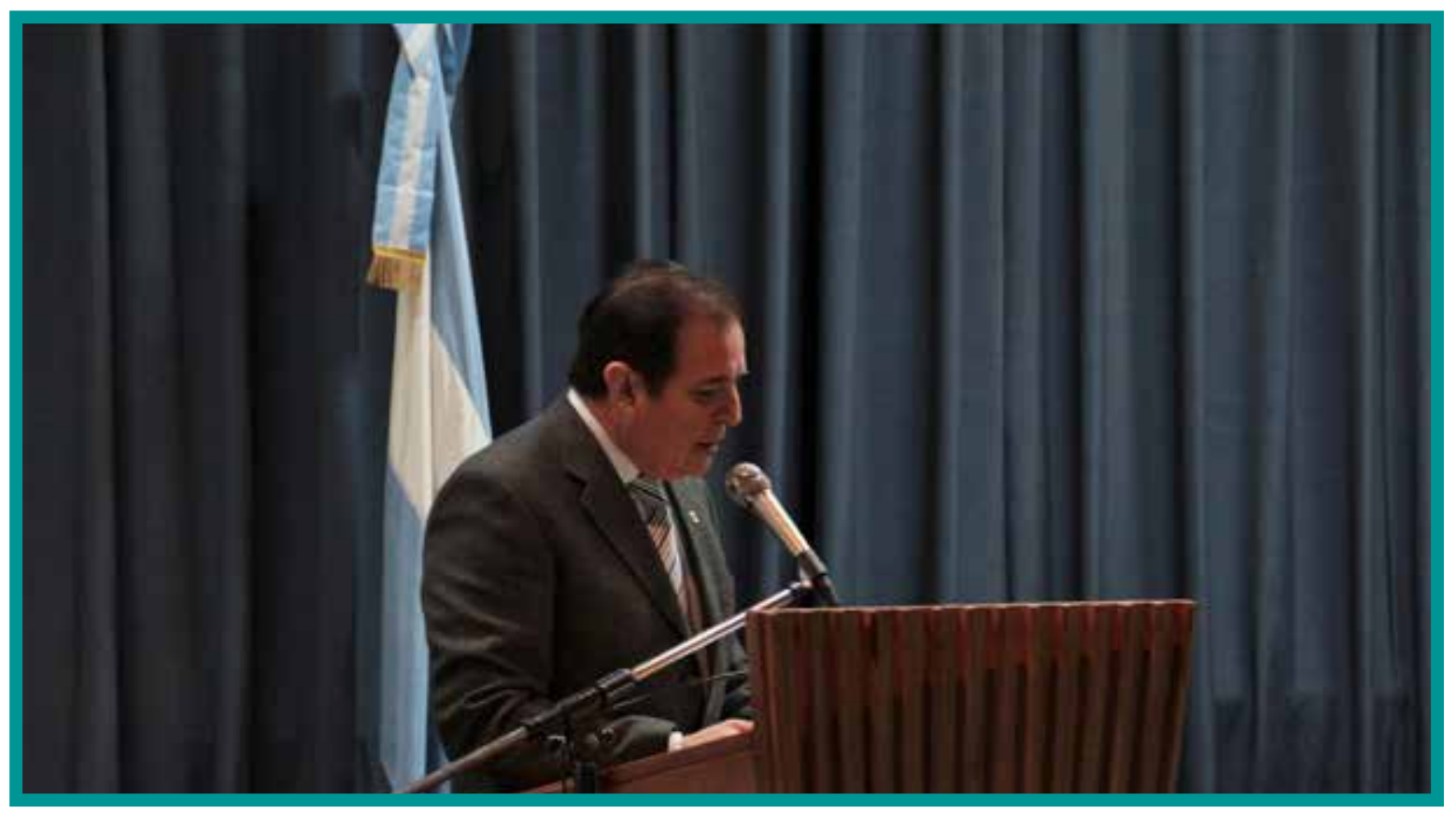

Con presencia de destacados investigadores y profesionales nacionales e internacionales, se inauguraron en la Facultad de Odontología un encuentro de becarios e investigadores que buscan difundir e intercambiar conocimientos y experiencias en el campo de la odontología. Se trata de la "IX Jornada de Ciencia y Tecnología", "VI Jornada Nacional de Becarios y Tesistas" y "I Jornadas Internacionales" organizadas por la Facultad de Odontología de la UNNE con el objetivo de generar un espacio de reflexión y debate sobre la ciencia y la tecnología, así como para fortalecer los vínculos científicos y tecnológicos entre las disciplinas afines a la Odontología.

Las jornadas, con asistentes de distintas provincias argentinas y de países vecinos, fueron oficialmente inauguradas con un acto con la presencia de la Rectora de la UNNE, profesora Ma- ría Delfina Veiravé, el Decano de la Facultad de Odontología, profesor Roque Oscar Rosende y la Secretaria de Investigación y Desarrollo de la facultad, doctora María Mercedes González.

Estuvieron además presentes la Secretaria General de Ciencia y Técnica de la UNNE, profesora María Silvia Leoni, el Secretario General de Vinculación y Transferencia, arquitecto Gustavo Tripaldi, Decanos, demás autoridades de Rectorado y de las distintas unidades académicas y centros científicos de la UNNE.

La Rectora de la UNNE destacó la confluencia de distintas jornadas en un mismo tiempo y espacio, que constituye un ámbito propicio para compartir experiencias de estudios y desarrollos tecnológicos, asociados al campo de la odontología.

"Este esfuerzo que implican las jornadas muestra la decisión institucional de la Facultad 


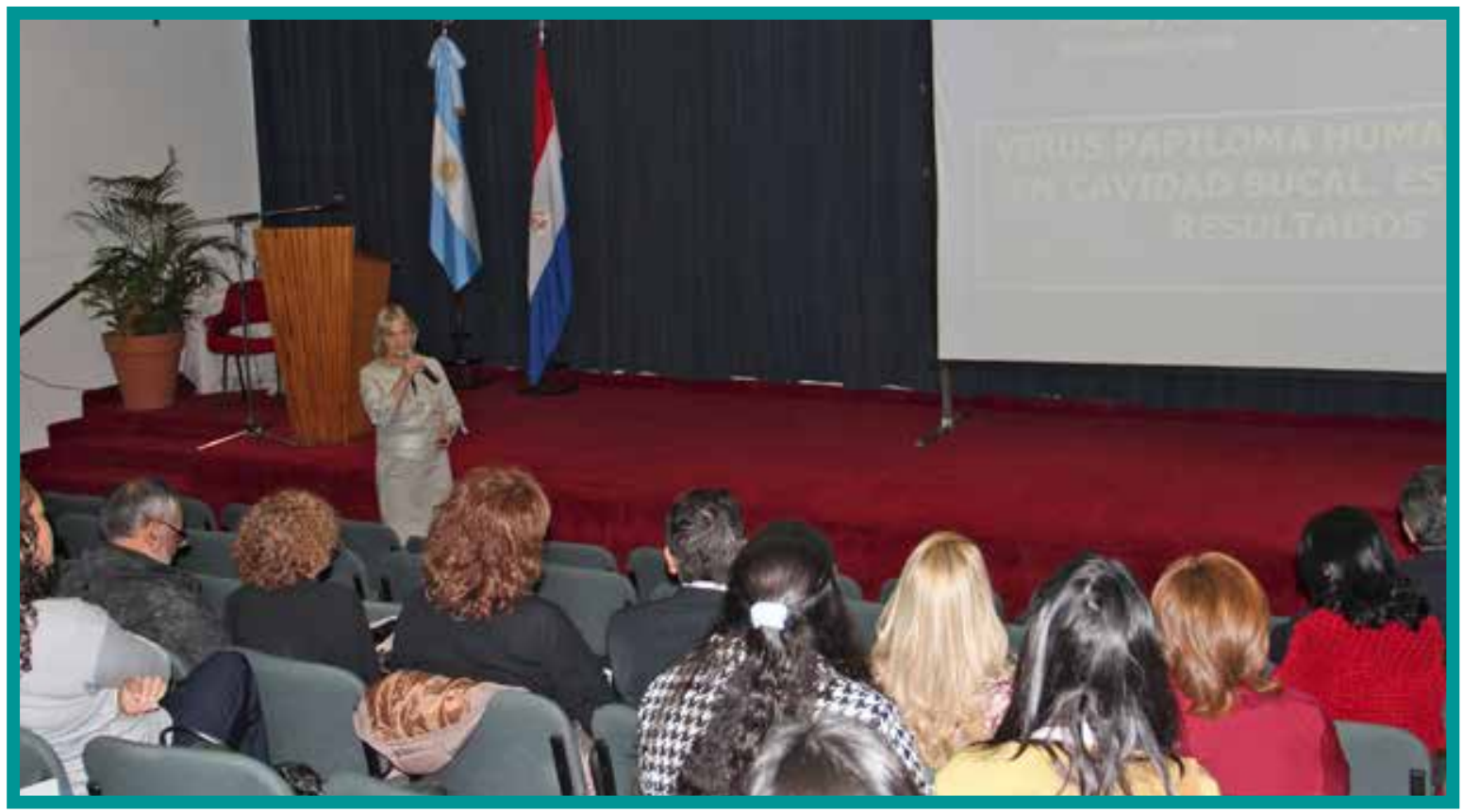

de Odontología de fortalecer vínculos, de reflexionar colectivamente sobre la ciencia, investigación e innovación" sostuvo.

"Adhiero a la continuidad de estos espacios de aprendizaje colectivo" remarcó en esa línea.

En particular sobre el campo de la odontología, señaló que compartir experiencias resulta sumamente importante a la hora de planificar estrategias y políticas que puedan contribuir, por ejemplo, en cuestiones tales como articular mejor la investigación con la extensión, la transferencia y los servicios a la comunidad.

Destacó la relevancia de aunar criterios en la identificación de áreas de vacancia, prioridades de la región, y el sostenimiento de la investigación áreas de grado y posgrado.

Señaló que la UNNE se encuentra trabajando en mejores articulaciones entre proyectos, pensando en líneas o programas comunes, desde perspectivas interdisciplinarias.

La Rectora llamó también a pensar cual es valor del conocimiento que generamos, pues el conocimiento es un bien social que debe estar

\section{"Este esfuerzo que} implican las jornadas muestra la decisión institucional de la Facultad de Odontología de fortalecer vínculos, de reflexionar colectivamente sobre la ciencia, investigación e innovación"

Rectora de la UNNE, profesora María Delfina Veiravé 


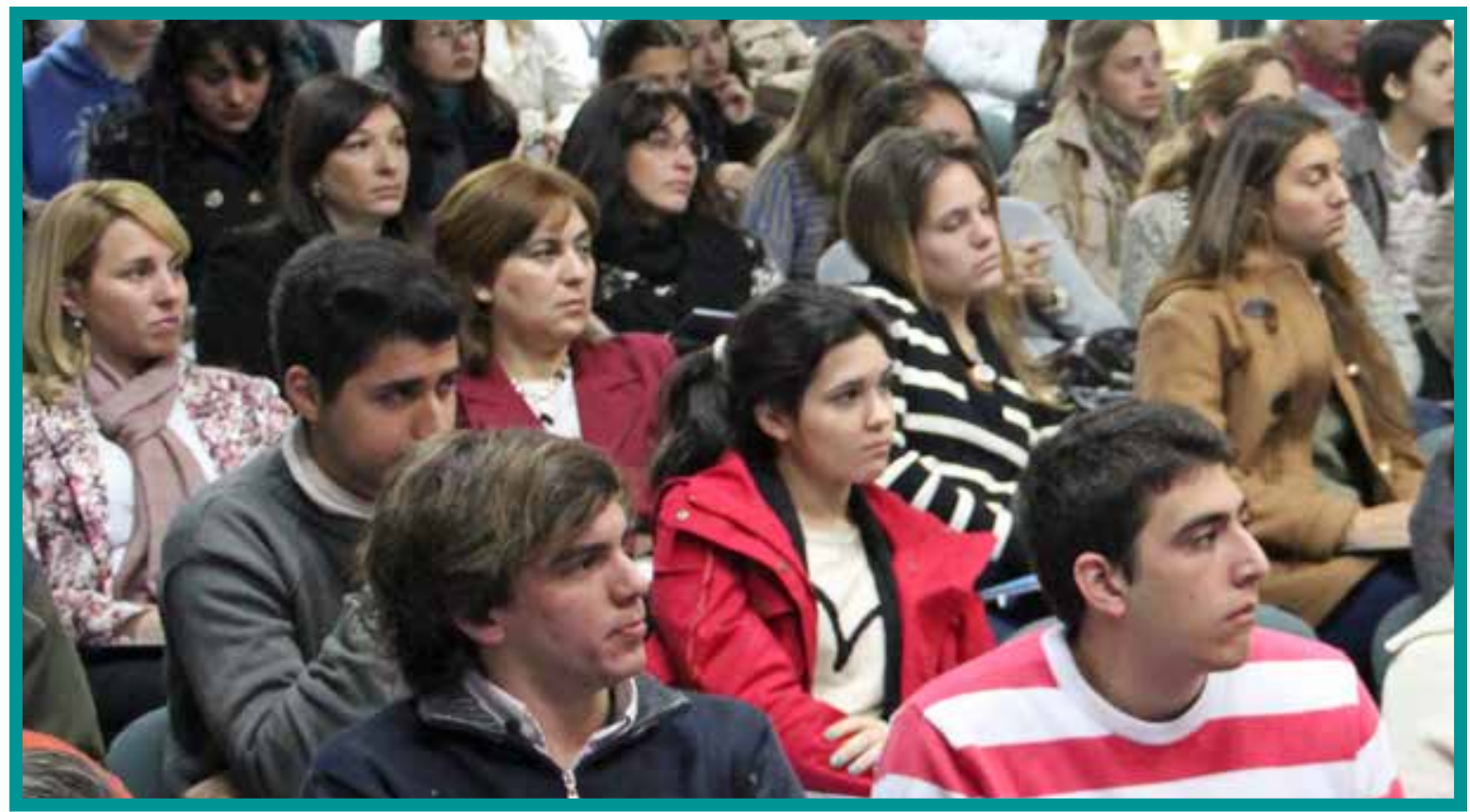

orientado prioritariamente a la resolución de problemas de la sociedad.

Por su parte, el Decano Roque Oscar Rosende remarco que la sociedad requiere capital humano para resolver sus problemas más inmediatos, y que contribuir a generar este capital es una de las misiones fundamentales de la universidad y la herramienta fundamental para lograrlo es la ciencia, "por eso es necesario cultivarla".

Expresó que las políticas en ciencia, tecnológica e innovación deben tener en cuenta el valor social del conocimiento y destacar la necesidad que los mismos se conviertan en acciones concretas en el contexto social.

Al respecto, sostuvo que constituye un lineamiento institucional de la Facultad de Odontología el desarrollo del espíritu científico en alumnos, docentes e investigadores, y así lo demuestra la fuerte impronta que llevan recorriendo desde 2006 a la fecha, "afianzando la investigación desde el grado al posgrado", como eje transversal en las actividades de la facultad, que no sólo promueva nuevo conocimiento sino que además pueda validarlo y transferirlo a la práctica profesional.

"Hemos cumplido el Plan de Desarrollo Institucional 2010-20I4 en cuanto a objetivos, acciones y metas propuestas. La función investigación nos encuentra con nuevos propósitos con el fin de fortalecer la continuidad del proyecto visionado" destacó el profesor Rosende.

Continuó que "nuestra meta es garantizar la formación de investigadores en las distintas áreas, que reafirmen la calidad académica y científica de nuestra facultad y de la universidad.

En tanto, la doctora María Mercedes González, a cargo de la Secretaría de Investigación y Desarrollo, señaló que la meta para la función investigación en la facultad es "no quedarnos sólo con lo realizado" sino seguir pensando, realizando en forma permanente y reorientando los caminos de la investigación con los cambios que de manera permanente se suceden en la disciplina. 


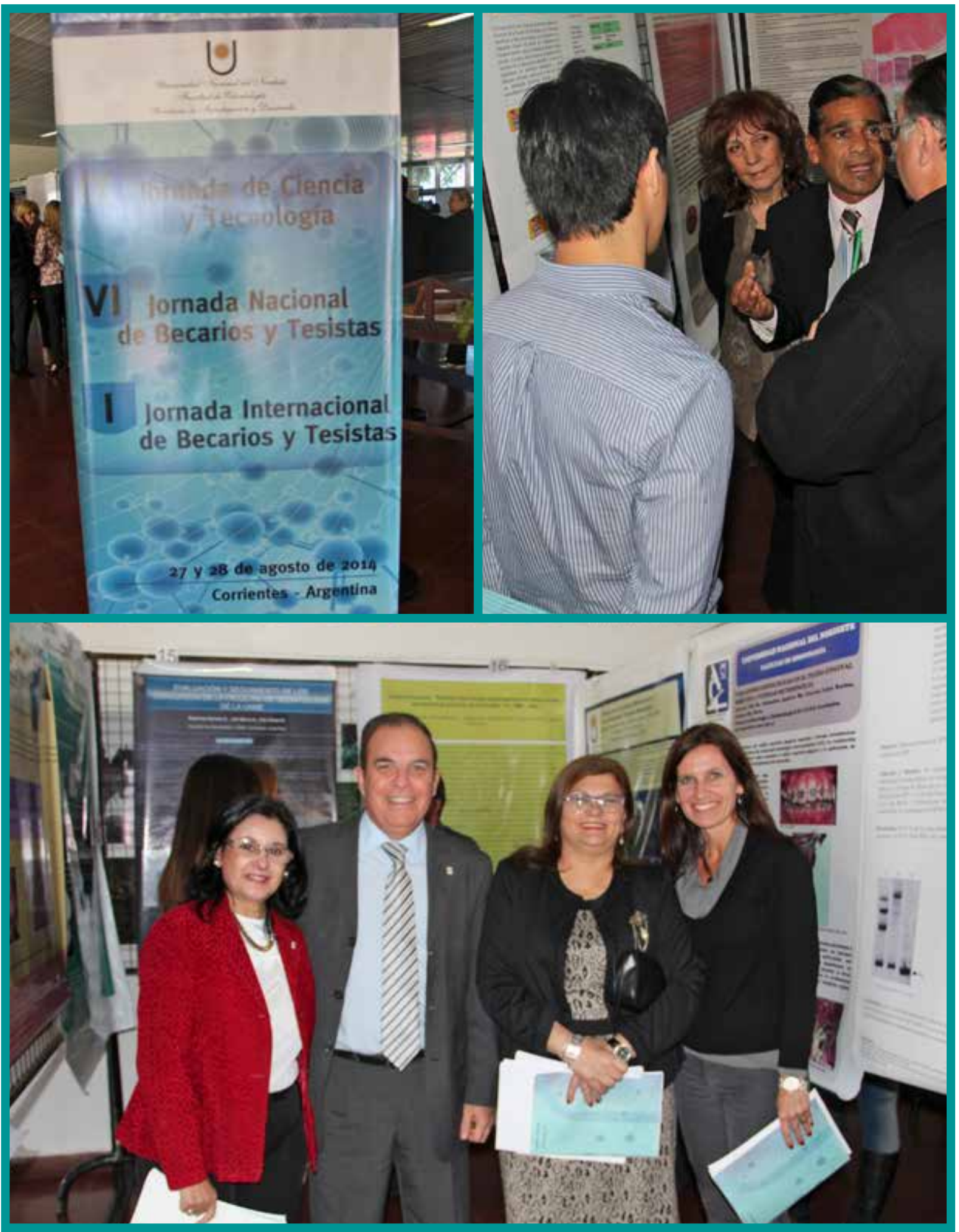




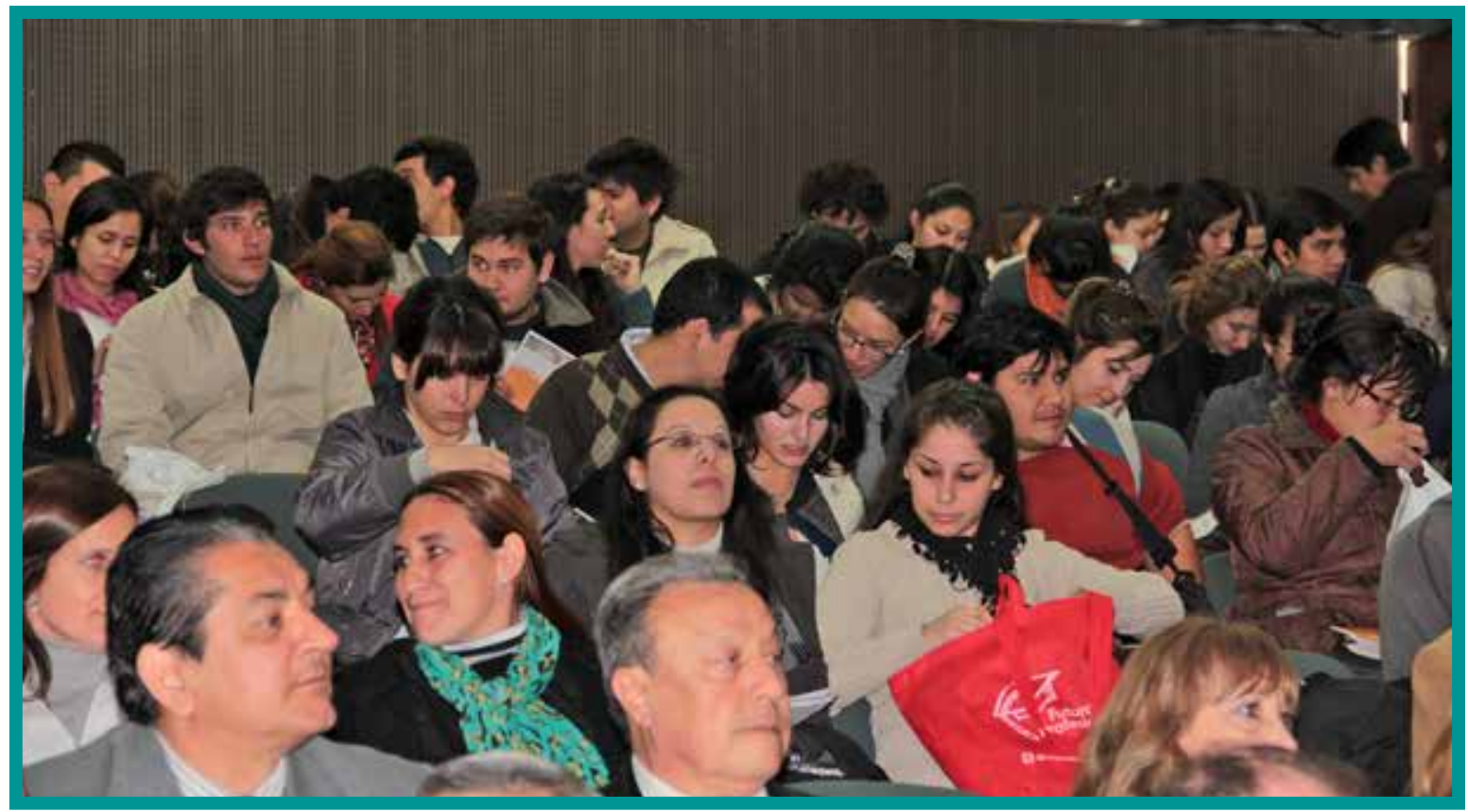

Remarcó, asimismo, que el verdadero cambio está en los actores, docentes, investigadores, becarios, que deben ser partícipes del accionar activo y colectivo, con una mirada amplia del proyecto institucional.

Explicó que la Facultad se propuso como objetivo consensuar entre sus miembros criterios significativos que aporten a la mejora de la investigación y el desarrollo, pero sin olvidar el contexto social.

La Secretaria de Investigación y Desarrollo de la Facultad de Odontología en particular instó a los becarios al esfuerzo para el desarrollo personal, pero sobre todo para preparase a fin de responder, desde la actividad científica, a las demandas sociales para la ciencia.

\section{Títulos de trabajos presentados}

Rol de la inmunoglobulina a salival como marcador biológico de la caries dental

Autores: Hormaechea, María I. - Martínez, Sandra E. - Juárez Rolando $P$.
Utilización de asociaciones no habituales para la irrigación del conducto radicular Autores: Lugo de Langhe, Carlos Daniel - González, María Mercedes

Comparación de dos selladores de fosas y fisuras aplicados en pacientes con discapacidad motora

Autores: Encina Tutuy, Alejandro J - Martínez, Sandra E. - Vila, Vilma G.

Acción térmica del mate asociada a la producción de cáncer bucal

Autores: Fernández, Estefanía R. - González, María Mercedes.

Determinación del consumo frecuente de mate como factor predisponente de lesiones precursoras

Autores: Od. Rosales Carlos Alejandro, Dra. Maria Mercedes Gonzalez

Aislamiento del lactobacillus spp de caries para prueba de sensibilidad ante propóleos de corrientes

Autores: Ramírez Ayala, Nicelly- Rosende, Roque Oscar- Vaculik, Patricia Alejandra- Lozina, Laura. 


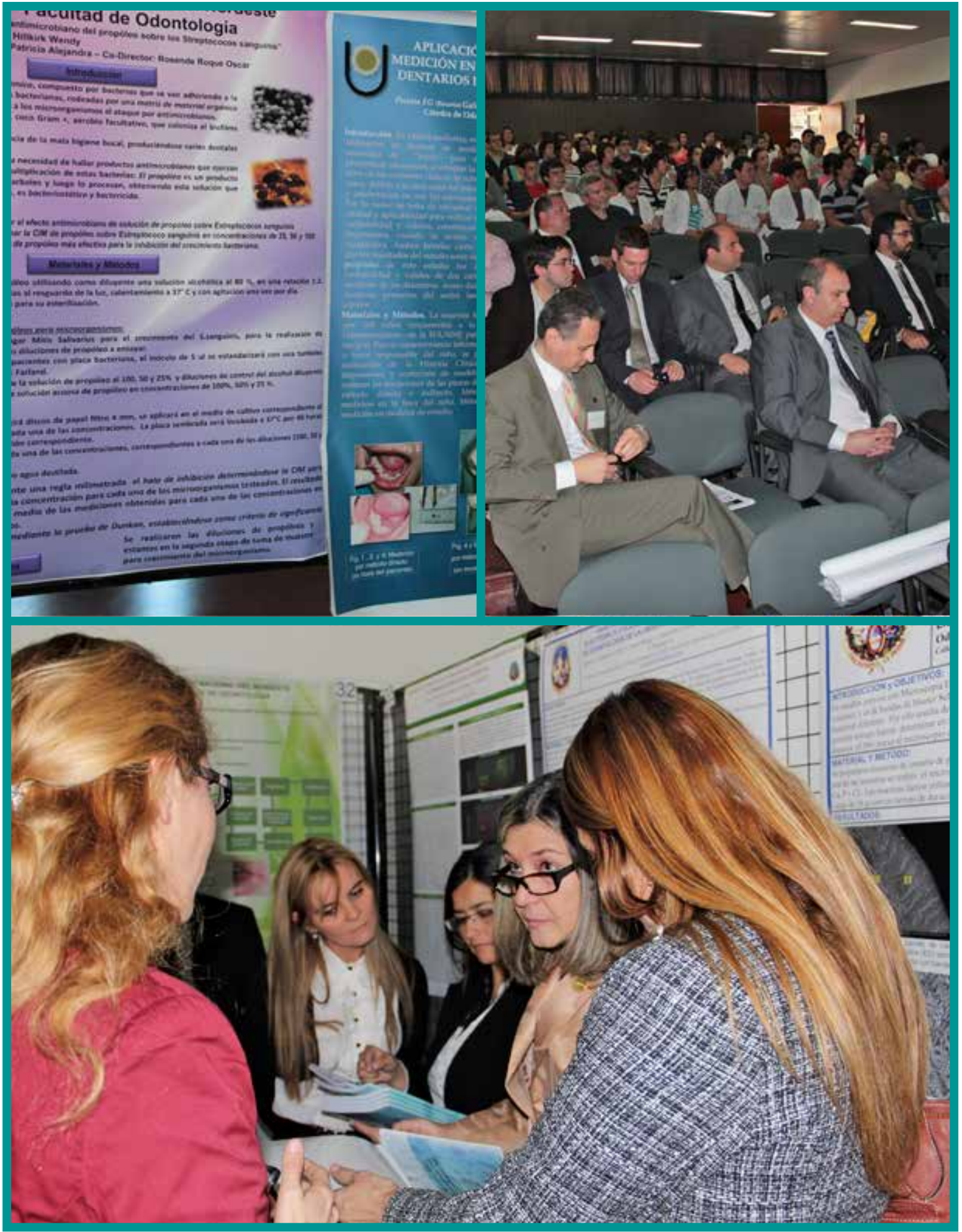


Efecto del consumo de tabaco sobre la Concentración de Fosfatasa Alcalina Salival Autores: Ledesma Facundo, Cuzziol Fernando, Celia Armando, Acuña Miguel, Juárez Rolando Pablo.

Marcadores de remodelamiento óseo en pacientes con enfermedad periodontal

Autores Domínguez Machado, Silvana Andrea; Celia, Armando; Acuña, Miguel Jorge; Juarez, Rolando Pablo Expresión de E-cadherina y Factor de crecimiento epidérmico en leucoplasias orales

Autores Od. Fortin Pedro; Mgter. Morales Sergio; Mgter. Briend María

Esmalte y Dentina, Histomorfologia de la Atricion en Piezas Dentarias Temporarias

Autores Lezcano Melisa Raquel, Gili Ma. Alejandra, Zamudio Ma. Eugenia

Cambios en los tejidos duros de dientes con atrición

Autores: Od. Solis Arce Elena P - Mgter. Zamudio María E- Od. Gili Ma. A.

Percepción del Consentimiento Informado en Odontólogos y Pacientes de Corrientes Capital

Autores: Marasso Spaciuk, Natalia Inés, Ariasgago, Olga Leonor

Efectividad del barniz de clorhexidina I\% en Streptococos mutans en primeros molares permanentes.

Autores: Od. Noelia E. Perez Quintana- Mgter Vilma G. Vila.- Mgter. Andrea V. Galiana

Disposición e incidencia de los conductos mesiales de los primeros y segundos molares inferiores

Autores: Fernado Arce, Vivian Benitez, Maria Caballero, Melisa Preda, Natalia Prieto

Patologías de la cavidad bucal asociadas con enfermedades parasitarias

Autores: Ramirez, L., Karaben, V., Rea M.

Técnicas de examen visual y DIAGNOdent para detección de caries incipiente en superficies oclusales.

Autores: Falcón, Diana E.; Valdovinos Zaputovich, Bertha M.
Hábitos de Salud Bucodental en relación al Nivel Socioeconómico en Individuos Adultos Autores: Dho, María Silvina Valoración de Salud Bucal en Infantes luego de la Aplicación de un Programa Preventivo. Autores: Molina Casco, Maria Cecilia. Cardozo, Juana Beatriz. Perez, Rita Silvia.

Efecto de Infusiones de llex - Paraguariensis sobre la Concentración de Fosfatasa Alcalina Salival.

Autores: Ponce Jorge O, Celia Armando C, Acuña Miguel J, Juárez Rolando $P$.

Prácticas de autoatención ante problemas buco-dentales en pobladores de los Esteros del lberá

Autores: Rea, Ana E. - Karaben, Viviana E. - Zamudio, María E.

Criterios clínicos para el diagnóstico de la Disfunción Temporomandibular

Autores: Edgardo Daniel Acevedo (alumno becario) Dra. María Julia López Vallejos (directora) Dr. Eduardo Buffil (co-director).

Relación de la Vertical Pterigoidea con el hueso hioides en pac. con la convexidad facial reducida

Autores: Od. Lorena Y. Palczikowski, Mgter. Carmen I. Collante de Benítez.

Prevalencia de apiñamiento dentario anterior en pacientes del hospital odontológico de la FOUNNE

Autores: Lohrman Cristal D; Peláez Alina N; Mazza Silvia $M$.

Alteraciones de oclusión en sentido transversal en pacientes del hospital odontológico de Ia FOUNNE

Autores: Oivera Paola B; Peláez Alina N; Mazza Silvia $M$.

Interacción "in vitro" de bacterias cariogénicas y bacterias de origen alimentario

Autores: SIn Cynthya C, Vasek Olga M, Ortega Silvia M Detección en saliva de lactato deshidrogenasa como biomarcador de lesión gingival Autores: Director de Beca: Monzón, Elpidio Javier Becaria de pregrado: Naumovich Gauna, María de los Angeles 
Estudio comparativo de Índice de Criterios Diagnóstico de TTM e Índice de Disfunción de Helkimo

Autores: Bernachea, Luciana Maricel. González, María Mercedez. López Vallejos, María Julia

Estudio del ceod en niños del Jardín Infantes $N^{\circ} 7$ Pinocho de Corrientes Capital

Autores: von Steiger, Francisco Germán; Cardozo, Beatriz Juana; Silvia, Rita Perez; Vaculik, Patricia.

Efectos antimicrobianos del propóleo sobre los Estreptococos sanguinis

Autores: Becaria:Hillkirk Wendy Director: Od. Vaculik, Patricia Alejandra Co- Director: Prof. Rosende, Roque Oscar

Comportamiento difusional in vitro de los iones oxhidrilo y calcio de Fórmulas magistrales de hidróxido de calcio con vehículo gel de aloe vera

Autores: Montiel Natalia B- Rocha Maria T.- Avalos Llano Karina R.- Lozina Laura

Relación del pH salival y lesiones de caries cervicales en pacientes con Síndrome de Down

Autores: Pochettino, Macarena. Vila, Vilma. Martinez Sandra. Barrios, Carolina

Diagnosticar por medios citológicos la presencia de Cándida en pacientes portadores de prótesis en Cátedras Clínicas de la Facultad de Odontología de la Universidad Nacional del Nordeste

Autores: Tascón Valeria Carolina- Almirón María Silvia- Montenegro María de las Angustias

Eficacia de propóleos de la región de Corrientes frente el Actinomices

Autores: Taube Henry Luis- Vaculik Patricia Alejandra- Rosende Roque Oscar

Conocimientos sobre caries dental e inflamación gingival que presentan padres/tutores de niños en edad preescolar

Autores: Flores, Ana Lucía; Cardozo, Beatriz Juana; Dho, María Silvina

Efectos antimicrobianos del propóleo sobre los Estreptococos sanguinis

Autores: Hillkirk Wendy Od. Esp. Vaculik Patricia, Alejandra Prof. Mter. Rosende Roque, Oscar

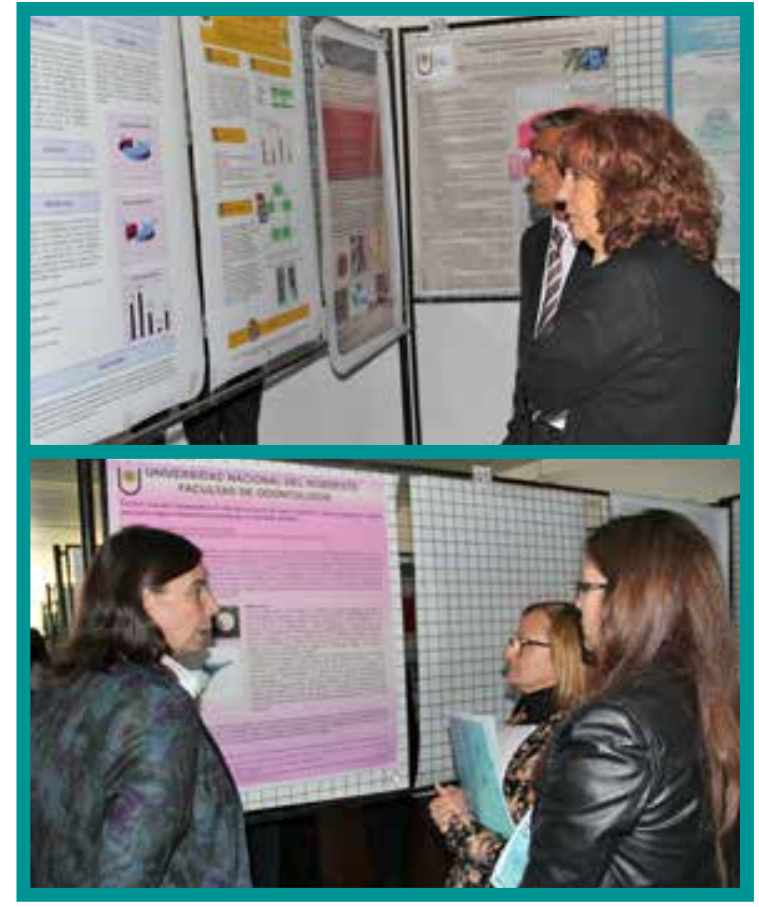

Actividad microbiana in vitro de la Stevia Rebaudiana Bertoni sobre el estreptococo mutans

Autores: Nazarena Rodríguez Vigay; Cardozo Beatriz; Silvia Perez

Problemática y conflictiva derivada de la viabilidad del contrato entre cónyuges en la empresa familiar

Autores: Henderson, María A. - Casella, Aldo P. - Vallejos, César A.

Estudio clínico de disfunción Temporomandibular en pacientes desdentados totales

Autores: Gómez Valeria, Huber Laura, Zamudio María Eugenia, Altamirano Mercedes.

Estudio in vitro de la fuerza de unión de una Resina Reforzada, utilizando dos Sistemas de tratamiento en la superficie dentaria

Autores: Pereyra Coimbra, Ángeles; Zamudio, María E.; Álvarez, Nilda; Christiani, Juan J.

Estudio in vitro de la resistencia al cizallamiento de una resina reforzada según el tratamiento del sustrato

Autores: Rodríguez Angelina; Zamudio, María E.; Álvarez, Nilda; Christiani, Juan J. 